\title{
Simulation of Optical Transmission System to Compensate Dispersion Using Chirped Fiber Bragg Grating (FBG)
}

\author{
Manpreet Kaur ${ }^{1}$, Himali Sarangal ${ }^{2}$ \\ Student (M.Tech), Department of Electronics and Communication Eng. GNDU Regional Campus Jalandhar, India \\ Asst.Prof, Department of Electronics and Communication Engineering GNDU Regional Campus Jalandhar, India ${ }^{2}$
}

\begin{abstract}
Fiber Bragg Grating (FBG) is one of the applicable and important components in optical communication system. In this paper, chirped FBG has been studied as a dispersion compensator in an optical communication system for the different lengths of grating and apodization functions. All the simulations are done in OPTISYSTEM 7.0 simulation software at $10 \mathrm{Gbits} / \mathrm{sec}$ and $210 \mathrm{~km}$ of transmission fiber. The simulated transmission system has been analyzed on the basic of different parameters, which are BER, Q-factor, Output power and Eye height.
\end{abstract}

Keywords: Fiber Bragg Grating (FBG), eye diagram, BER and Q-factor.

\section{INTRODUCTION}

Optical communication is a system transmitting information in form of light through an optical fiber. The light acts as an electromagnetic carrier wave that is modulated to carry information. The basic optical communication system consists of three elements which are light source that convert electrical signal into optical signal, optical fiber which acts as a transmission medium and photo detector or light detector that converts the optical signal into electrical signal at the receiver side. The goal of every communication system is to increase the transmission distance and speed. Like other communication systems the optical communication systems also faces problems such as dispersion, attenuation, losses and non-linear effects, which degrade its performance. Among them the dispersion affects the system most and it is difficult to overcome as compared to other losses. Thus it is important to incorporate an effective dispersion compensation technique in optical communication systems that lead to performance enhancement of the transmission system. [1]

The optical amplifiers (EDFA, SOA etc) has resolved the problem of optical fiber losses and made the long distance transmission possible without electronic regenerators. But the dispersion and other non-linearities of optical fibers hindered ultra-long distance transmission and high bit rate transmission. Dispersion is defined as the pulse spreading in an optical fiber. When different wavelengths of light pulses are launched into the optical fiber, these pulses travelled with different speeds due to the variation of refractive index with wavelengths. The light pulses tend to spread out in time domain after travelling some distance in fiber and this is continued throughout the fiber length. This phenomenon is known as dispersion. Since each pulse spreads and overlap with its neighbouring pulse, becoming indistinguishable at the receiver end. This effect is known as inter symbol interference (ISI). Dispersion limits the information carrying capacity at high transmission speeds, reduces the effective bandwidth and increases the bit error rate (BER). In single mode fiber (SMF), the performance is primarily limited by chromatic dispersion (CD) and polarization mode dispersion (PMD). CD occurs because of the wavelength dependency of refractive index of fiber and the fiber has some inherent properties like birefringence that lead to PMD. [2]

In order to improve the overall system performance affected by dispersion, several dispersion compensation techniques are proposed and analyzed. Among the various techniques proposed in literature, the most suitable techniques are: Dispersion compensating fibers (FBG), Fiber bragg gratings (FBG), Electronic dispersion compensation (EDC), Digital filters and Higher order mode (HOM) fibers.

In this paper, the FBG is used as the dispersion compensator. The rest of the paper is organized as followed: In section II the FBG operation principle is described. In section III, simulation of transmission system is presented. Section IV presents the results and analysis and section $\mathrm{V}$ concludes the paper.

\section{FibER BRAGG GRATING (FBG) OPERATION PRINCIPLE}

One of the most advanced techniques being used in the dispersion compensation methods is FBG. FBG is a piece of optical fiber with the periodic variation of refractive index along the fiber axis. This phase grating acts like a band rejection filter reflecting wavelengths that satisfy the Bragg condition and transmitting the other wavelengths. The reflected wavelength changes with grating period. Thus, FBG is very simple and low cost filter for wavelength selection that improves the quality and diminishes the costs in optical networks. [3] The equation relating the grating periodicity, Bragg wavelength and effective refractive index of the transmission medium is given by:

$$
\lambda_{B}=2 \mathrm{n} \Lambda
$$


In this equation, $\lambda_{B}, \mathrm{n}$ and $\Lambda$ are the bragg wavelength, refractive index of core and grating period respectively.

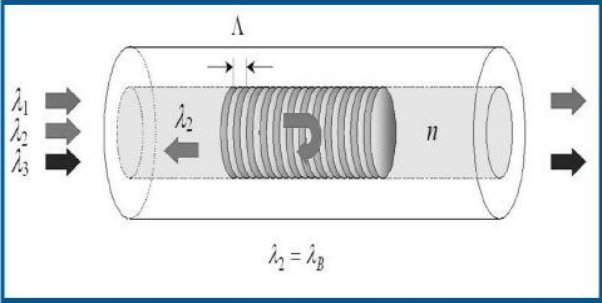

Fig. 1: Principle of FBG [4]

A chirp is variations in the grating period created along the FBG. As shown in Fig.2 when a signal enters into chirp, different wavelengths are reflected from different parts of grating. Thus, a delay related to the wavelength of the signal is produced by grating. [4]

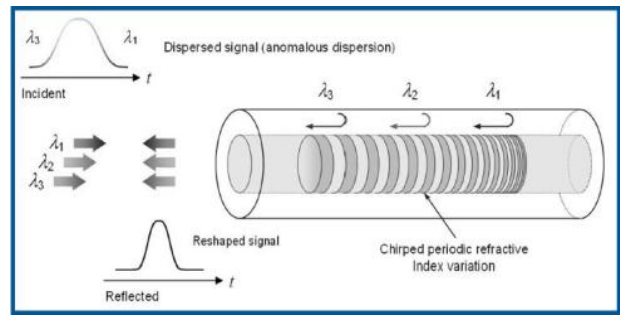

Fig. 2: A chirped FBG principle [4]

\section{METHODOLOGY}

All the simulations are done in OPTISYSTEM 7.0 simulator software. It is an advanced, innovative and powerful software simulator tool used for design, testing and optimization of virtually any type of optical link. [4]

We use the parameters in Table 1 in order to simulate the optical transmission system. The model of the simulated system is as shown in Fig.3. In the simulation, the transmitter section consists of data source, modulator driver (NRZ), laser source and Mach-Zehnder (M-Z) modulator. We use the continuous wave $(\mathrm{CW})$ laser with frequency $193.1 \mathrm{THz}$ and output power of $15 \mathrm{dbm}$, which is externally modulated at $10 \mathrm{Gbits} / \mathrm{sec}$ with a non-return to zero (NRZ) pseudo random binary sequence in a M-Z modulator with $30 \mathrm{db}$ extinction ratio. Two EDFAs are used as optical amplifiers in the system with gain of $40 \mathrm{db}$ and $10 \mathrm{db}$ with noise figure $4 \mathrm{db}$. The single mode fiber (SMF) of length $210 \mathrm{~km}$ is used as the transmission medium. And FBG is used as the dispersion compensator. At the receiver side, the PIN diode is used as a photo detector, which converts the optical signals into electrical, having $1 \mathrm{~A} / \mathrm{W}$ responsivity and $10 \mathrm{nA}$ of dark current. Then the electrical signal is filtered by low pass Bessel filter and $3 \mathrm{R}$ regenerator is used for regeneration.

Table 1. Simulation parameters

\begin{tabular}{|c|c|}
\hline Parameters & Value \\
\hline Dispersion $(\mathrm{ps} / \mathrm{nm} . \mathrm{km})$ & 17.25 \\
\hline Dispersion slope $\left(\mathrm{ps} / \mathrm{nm}^{2} / \mathrm{km}\right)$ & 0.085 \\
\hline Attenuation $(\mathrm{db} / \mathrm{km})$ & 0.2 \\
\hline Fiber length $(\mathrm{km})$ & 210 \\
\hline
\end{tabular}

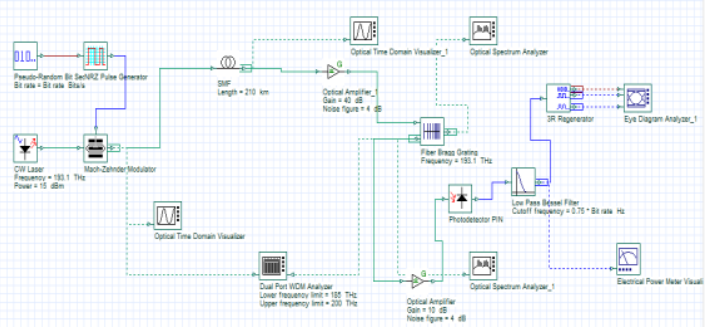

Fig. 3: Model of simulated system

\section{IV.RESULTS AND DISCUSSION}

Fig. 4(a) shows the eye diagram of modulator output that is the main information shape in fiber input. Fig. 4(b) shows the eye diagram of the fiber output that is affected by dispersion. We can see that the data before entering to fiber and after exit from the fiber are not same; this is the main disadvantage of communication system. Therefore, we use the FBG as the dispersion compensator after the optical fiber. Fig. 4(c) shows the eye diagram of the signal at the receiver end with dispersion compensation. The FBG parameters used are shown in Table 2.

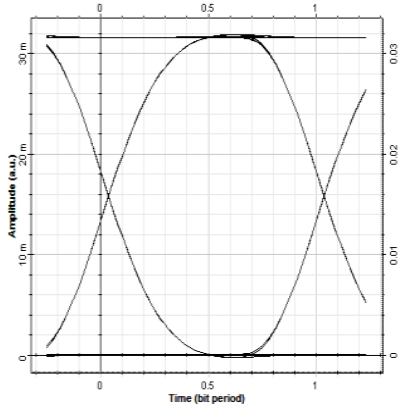

(a)

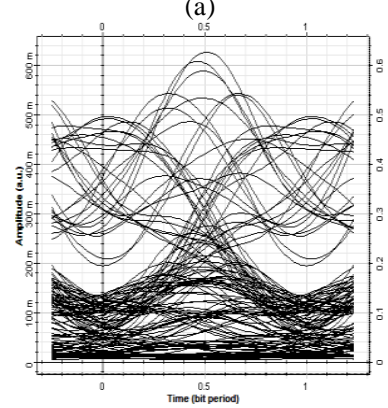

(b)

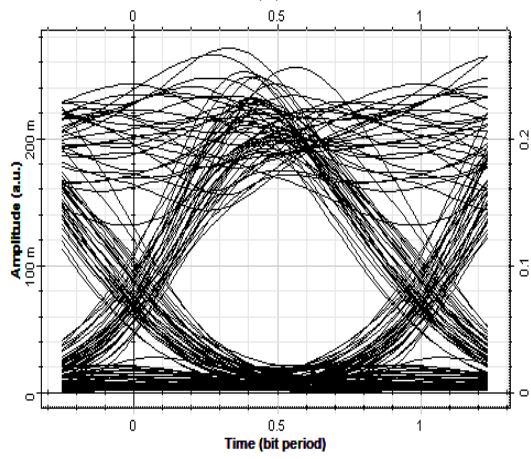

(c)

Fig. 4: (a) Eye diagram of the signal from M-Z modulator; (b) Eye diagram of the signal after propagating over $210 \mathrm{~km}$ without dispersion compensation and (c) Eye diagram with FBG as the dispersion compensator 
International Journal of Advanced Research in Computer and Communication Engineering Vol. 4, Issue 2, February 2015

Table 2. FBG parameters

\begin{tabular}{|c|c|}
\hline Parameters & Value \\
\hline Frequency (THz) & 193.1 \\
\hline Effective refractive index & 1.45 \\
\hline Length of grating (mm) & 80 \\
\hline Apodization function & Tanh \\
\hline Chirp function & Linear \\
\hline
\end{tabular}

We achieved the most proper grating length for the proposed model of $80 \mathrm{~mm}$ by try and error method. Eye diagrams for the different lengths of grating are shown in Fig.5 and the values of Q-factor and BER for the different lengths of grating are tabulated into Table 3.

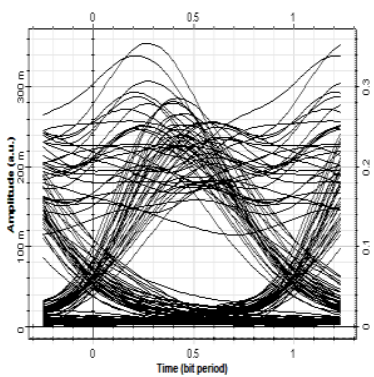

$75 \mathrm{~km}$

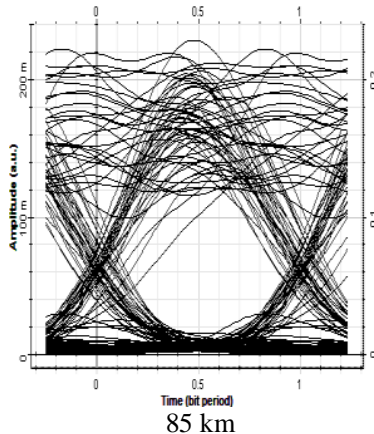

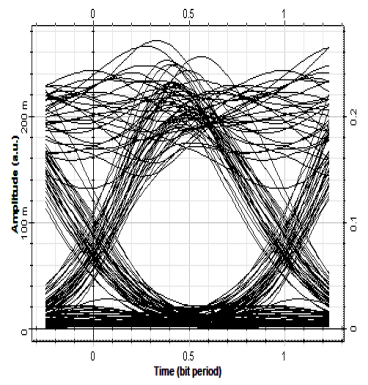

$80 \mathrm{~km}$

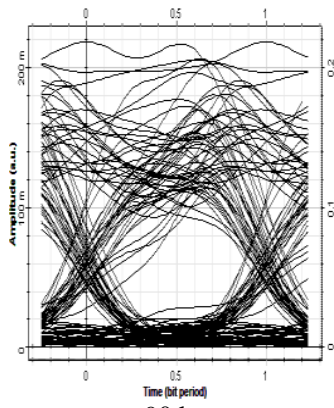

$90 \mathrm{~km}$
Fig. 5: Eye diagrams for the different grating lengths

Table 3. : Comparison of different FBG lengths

\begin{tabular}{|c|c|c|}
\hline $\begin{array}{c}\text { FBG length } \\
(\mathrm{mm})\end{array}$ & $\begin{array}{c}\text { Q-factor } \\
(\mathrm{db})\end{array}$ & BER \\
\hline 75 & 5.2124 & $7.16442 \mathrm{e}-008$ \\
\hline 80 & 8.65973 & $1.94706 \mathrm{e}-018$ \\
\hline 85 & 5.37153 & $2.53473 \mathrm{e}-008$ \\
\hline 90 & 3.84116 & $4.51004 \mathrm{e}-005$ \\
\hline
\end{tabular}

By comparing the values in Table 3 , we found that the 80 $\mathrm{mm}$ grating length is better for $210 \mathrm{~km}$ of optical fiber.

In Fig.6, the eye diagrams for different profiles of apodization depicted that Tanh and Gaussian give better results as compared to Uniform apodization function. The Q-factor and BER for the different apodization functions are tabulated into Table 4.

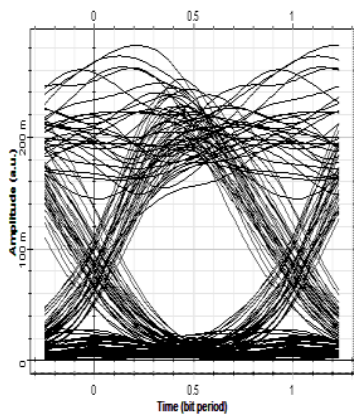

(a)

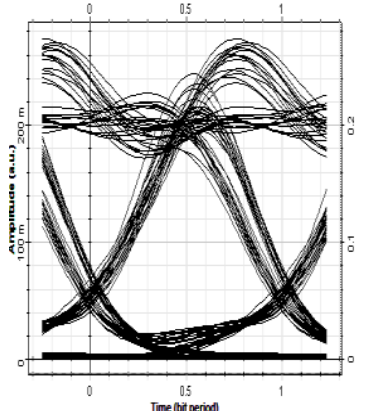

(b)

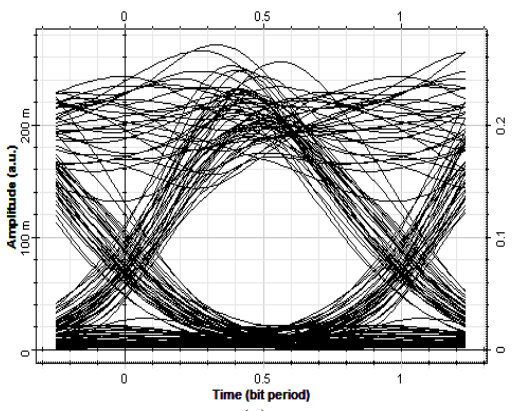

(c)

Fig. 6: Eye diagrams for different apodization functions: (a) Uniform (b) Gaussian and (c) Tanh

Table 4. Comparison of apodization functions

\begin{tabular}{|c|c|c|}
\hline $\begin{array}{c}\text { Apodization } \\
\text { function }\end{array}$ & $\begin{array}{c}\text { Q-factor } \\
(\mathrm{db})\end{array}$ & BER \\
\hline Uniform & 8.27338 & $5.38347 \mathrm{e}-017$ \\
\hline Gaussian & 10.5922 & $1.5591 \mathrm{e}-026$ \\
\hline Tanh & 8.65973 & $1.94706 \mathrm{e}-018$ \\
\hline
\end{tabular}

\section{CONCLUSION}

In this paper, we have simulated an optical transmission system. As soon as we observed dispersion, we decide to compensate it. For this purpose, we employed chirped FBG and simulate it. The system has been studied for the different lengths of grating and apodization functions. We have analyzed that the $80 \mathrm{~mm}$ grating length gives better results for $210 \mathrm{~km}$ of optical fiber at $10 \mathrm{Gbits} / \mathrm{sec}$.

\section{REFERENCES}

[1] M.A.Othman, M.M.Ismail, H.A.Sulaiman, M.H.Misran, M.A.Meor Said, Y.A.Rahim, A.N.Che Pee and M.R.Motsidi, An Analysis of 10 Gbits/sec Optical Transmission System using Fiber Bragg Grating (FBG), IOSR Journal of Engineering, ISSN: 2250-3021 Volume 2, Issue 7, July 2012, 55-61.

[2] Kaushal Kumar, A.K.Jaiswal, Mukesh Kumar and Nilesh Agrawal, Performance analysis of dispersion compensation using Fiber Bragg Grating (FBG) in Optical Communication, International Journal of Current Engineering and Technology, E-ISSN 2277-4106, P-ISSN 2347-5161, vol.4, No.3, June 2014, 1527-1531.

[3] Yogesh Chaba and R.S.Kaler, Comparison of various dispersion compensation techniques at high bit rates using CSRZ format, Elsevier ScienceDirect Optik 121, 2010, 813-817.

[4] S. O. Mohammadi, Saeed Mozaffari, and M. Mehdi Shahidi, Simulation of a transmission system to compensate dispersion in an optical fiber by chirp gratings, International journal of the Physical Sciences vol. 6(32), 2 Dec 2011, 7354-7360. 